\title{
Polipose adenomatosa familiar: um relato de caso
}

Sofia Correia,* Cátia Andrade**

\section{RESUMO}

Introdução: A síndroma de polipose adenomatosa familiar (PAF), na sua forma clássica, é autossómica dominante. Os indivíduos afetados podem permanecer assintomáticos por vários anos, pelo que se houver história familiar de PAF dever-se-á manter suspeição clínica. Assim, este relato de caso pretende reforçar o papel do médico de família na abordagem desta patologia rara e realçar a necessidade de um trabalho em equipa e de cuidados de saúde perfeitamente coordenados, tendo em conta as dificuldades existentes no seu diagnóstico atempado.

Descrição do caso: Utente com 20 anos observada pela sua médica de família por história de vómitos, dejeções diarreicas frequentes e perda ponderal de $7 \mathrm{~kg}$ com duas semanas de evolução. Ao exame objetivo, com dor à palpação e sinal de blumberg presente na transição da região peri-umbilical para o hipocôndrio esquerdo. Através da entrevista clínica e realização do genograma familiar verificou-se a existência de um padrão de transmissão hereditária de patologia do trato gastrointestinal. Foi enviada para o serviço de urgência hospitalar, onde se excluiu a existência de patologia cirúrgica abdominal e/ou ginecológica, tendo tido alta referenciada para consulta de medicina interna. Após realização de colonoscopia total ficou internada para resseção cirúrgica de adenocarcinoma do cólon sigmóide.

Comentário: Este caso realça simultaneamente a importância do genograma familiar e a limitação temporal à sua aplicação pelo médico de família. Reforça ainda a importância da integração e coordenação de cuidados de saúde nesta patologia. O médico de família deverá conhecer as possíveis complicações da intervenção cirúrgica realizada, bem como outras patologias associadas à PAF e qual o tipo de acompanhamento que deve ser feito nestes utentes. Neste caso há ainda que ter em consideração a necessidade de rastreio de outros familiares afetados e a importância da consulta pré-concecional e/ou de diagnóstico pré-natal.

Palavras-chave: Polipose adenomatosa familiar; Dor abdominal; Genograma; Medicina geral.

\section{INTRODUÇÃO}

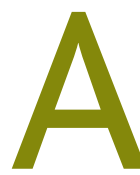
síndroma de polipose adenomatosa familiar (PAF) é caracterizada pelo desenvolvimento de centenas a milhares de adenomas colorretais. É acompanhada pelo aparecimento de cancro colorrectal em quase $100 \%$ das pessoas afetadas até aos 40 anos de idade, representando cerca de $0,5 \%$ dos casos de cancro colorretal. Na sua forma clássica é uma doença autossómica dominante.

Por ser habitualmente assintomática até ocorrer a transformação neoplásica, o seu diagnóstico precoce pode ser difícil no caso de não haver casos detetados. Assim, o conhecimento da história familiar permite um maior grau de suspeição, sendo fulcral que assim que

*Médica Especialista em Medicina Geral e Familiar, Unidade de Saúde de Itha de São Miguel, Açores.

**Médica Interna em Medicina Geral e Familiar, Unidade de Saúde de Ilha de São Miguel, Açores. seja feito o diagnóstico a um indivíduo se contactem todos os familiares que possam ser afetados para rastreio. ${ }^{2}$

O presente relato de caso pretende demonstrar a importância do trabalho em equipa na facilitação do acesso aos cuidados de saúde e na satisfação das necessidades em saúde dos utentes. Descreve uma patologia rara, na qual o genograma familiar tem um papel fundamental, exemplificando as dificuldades que, por vezes, existem no diagnóstico atempado e demonstra o papel do médico de família na abordagem de quadros indiferenciados e na coordenação de cuidados. Pretende-se ainda responder à questão de qual será o papel do médico de família no diagnóstico e acompanhamento desta patologia rara.

\section{DESCRIÇÃO DO CASO}

Identificação e história pessoal

Maria (nome fictício) é uma utente de 20 anos de 
idade, caucasiana, natural de São Miguel, onde sempre residiu. Tem o $12^{\circ}$ ano de escolaridade completo e trabalha como empregada de comércio. É solteira e sem companheiro no momento desta consulta.

Como antecedentes pessoais apresenta asma, rinite alérgica, miopia e astigmatismo. É acompanhada em consulta de cirurgia geral após exérese de fibroadenoma mamário. A sua medicação habitual inclui: contraceção oral combinada, inalador pressurizado de salbutamol 100 microgramas (duas inalações a cada oito horas nas crises) e inalador de pó contendo 320 microgramas de budesonida e 9 microgramas de formoterol (uma inalação de 12 horas em 12 horas).

\section{Caracterização familiar}

A Maria integra uma família nuclear em fase $V$ do ciclo familiar de Duvall e de classe social IV (média-baixa), de acordo com a escala de Graffar.

O genograma familiar e a psicofigura de Mitchell encontram-se representados na Figura 1, de acordo com as informações fornecidas pela Maria em consulta. Relativamente à história familiar destaca-se avó paterna falecida aos 57 anos por neoplasia intestinal, tia paterna com história de exérese de parte do intestino, duas primas paternas em primeiro grau com neoplasia intestinal, avô materno, mãe e duas tias maternas cegas por doença oftalmológica hereditária.

\section{História da doença atual}

No final de julho de 2016 Maria recorre à unidade de saúde para vacinação. Ao conversar com a enfermeira de família refere vários episódios de diarreia, vómitos e perda de peso acentuada. Perante este quadro, a enfermeira de família alertou a médica.

Maria foi observada no mesmo dia, referindo na consulta que se encontrava com vómitos alimentares (aproximadamente três a quatro por dia), não biliosos, não hemáticos e não fecaloides desde há duas semanas, com dejeções diarreicas de duas em duas horas com fezes sem sangue, muco ou pús e perda de peso de $7 \mathrm{~kg}$ (passou de $66 \mathrm{~kg}$ para $59 \mathrm{~kg}$ ) no referido período. Referia também anorexia, tonturas, dor abdominal tipo "agulha" (sic), intermitente, no hipocôndrio esquerdo e região peri-umbilical, sem irradiação, que aliviava com as dejeções, agravava com os vómitos e a tosse, acordando-a durante a noite. Referia ainda que sentia "um novelo" (sic) na transição da região peri-umbilical para o hipocôndrio esquerdo. Negava febre, dispneia, dor torácica e sintomas do trato genitourinário, como disúria, piúria, hematúria, urgência miccional, leucorreia ou hemorragia uterina anómala. Mencionava também obstrução nasal, rinorreia anterior mucosa, crises de esternutos e prurido nasal e do palato. Negava crises de asma no último mês.

Ao exame objetivo apresentava pele e mucosas coradas e hidratadas, sem sinal da prega cutânea. Tinha uma pressão arterial de 108/66mmHg, frequência cardíaca de 68 batimentos por minuto e saturação de oxigénio de 99\%. A auscultação cardíaca era rítmica, sem sopros, e na auscultação pulmonar apresentava murmúrio vesicular mantido e simétrico, com vários sibilos bilaterais expiratórios. O abdómen era normal à inspeção e à auscultação os ruídos hidroaéreos estavam presentes, com timbre normal e frequência aumentada. Sem massas ou organomegalias palpáveis apresentava dor à palpação superficial e profunda da região peri-umbilical, hipocôndrio e flanco esquerdos, com defesa e sinal de blumberg presente. Quando se encontrava em posição ortostática era possível palpar massa na transição da região peri-umbilical para o hipocôndrio esquerdo, dolorosa ao toque. O teste de gravidez era negativo e o exame com fita teste urinária apresentava apenas vestígios de leucócitos.

Posto isto, colocaram-se como hipóteses diagnósticas doença inflamatória intestinal, hérnia da parede abdominal encarcerada ou patologia hereditária do trato gastrointestinal, pelo que foi referenciada ao serviço de urgência do hospital de referência, após prescrição de medicação de acordo com queixas respiratórias. Foi ainda revista a técnica inalatória e feitas as devidas correções, uma vez que se objetivaram falhas na mesma.

No mesmo dia foi observada no serviço de urgência do hospital de referência pela cirurgia geral, tendo feito análises ao sangue e urina, bem como radiografia abdominal que se encontravam normais. Foi igualmente observada no serviço de ginecologia, tendo realizado ecografia pélvica que não revelou alterações.

Após excluírem uma possível causa cirúrgica e/ou ginecológica foi encaminhada para a área médica do serviço de urgência, onde teve alta após referenciação para consulta de medicina interna para estudo do quadro.

Em setembro de 2016 é observada em consulta de medicina interna. Na informação clínica de retorno 


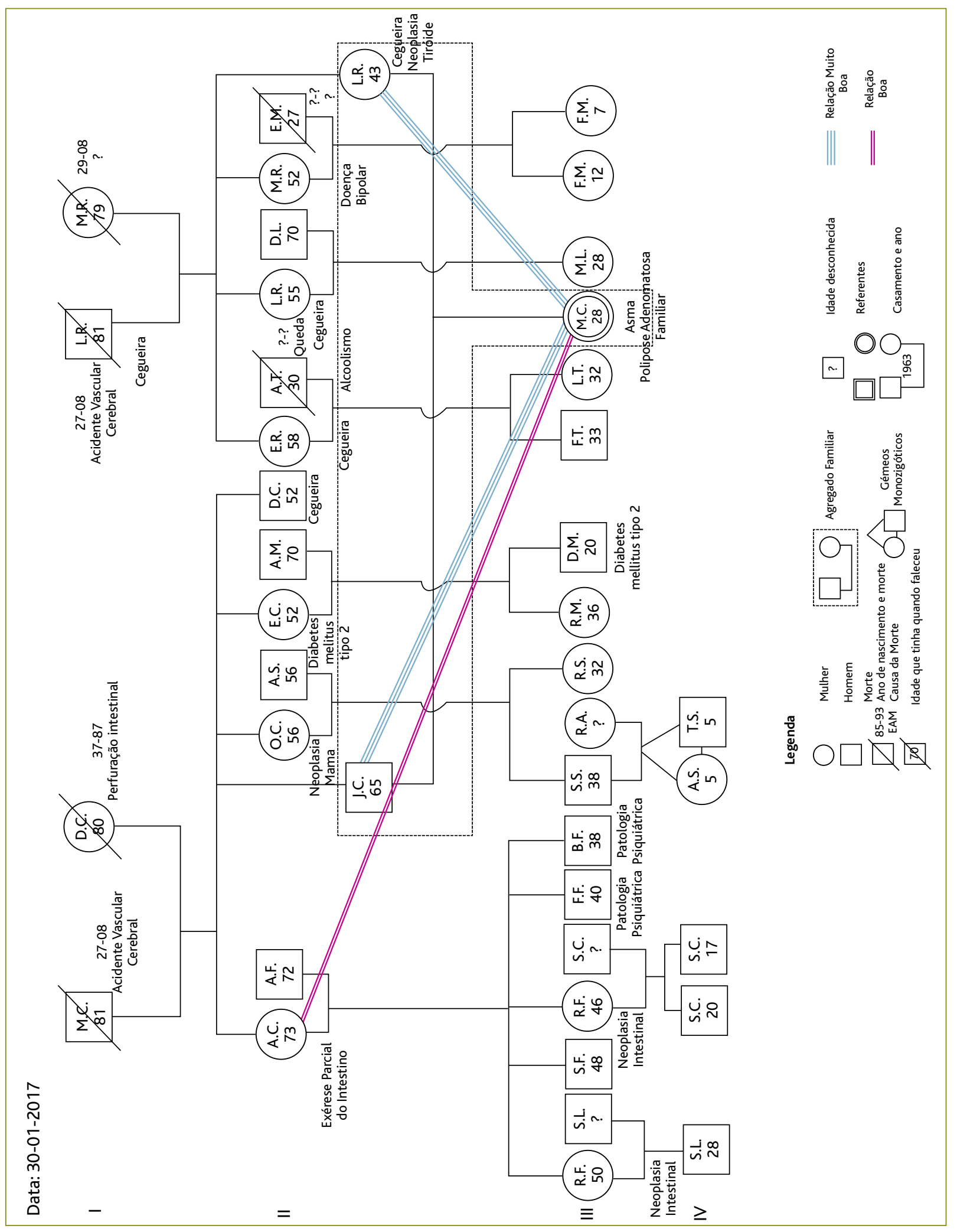

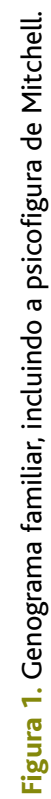


para a médica de família é referido que, apesar de parecer haver relação entre o início dos sintomas e problemas laborais e insónia, a utente relacionava a perda de peso apenas com a diarreia e com os vómitos. Mesmo não conseguindo palpar qualquer massa abdominal foi pedida uma ecografia abdominal para exclusão de hérnia da linha branca, o que não se verificou. Analiticamente, o hemograma, leucograma, proteína $\mathrm{C}$ reativa, função hepática e função renal eram normais, tendo sido pedida também a velocidade de sedimentação, função tiroideia, estudo da cinética de ferro, endoscopia digestiva alta e colonoscopia total. Foi medicada com trimebutina 200mg um comprimido três vezes ao dia e metoclopramida 10mg um comprimido duas vezes ao dia.

No início de outubro a utente é internada no serviço de cirurgia geral após realização de colonoscopia total, que mostrou massa no cólon sigmoide, vegetante e estenosante, cuja biópsia revelou adenocarcinoma do cólon sigmoide. A 14 de outubro foi submetida a colectomia total com anastomose íleo-retal baixa com bolsa em J, com disseção de todo o cólon por laparoscopia e isolamento do reto e anastomose íleo-retal por via laparotómica. A análise histológica revelou adenocarcinoma invasor, com áreas produtoras de muco, G2, que invadia toda a espessura da parede e o tecido adiposo subseroso, com serosa visceral poupada e mais de 100 pólipos no restante cólon. Da avaliação efetuada concluiu-se que apresentava um tumor em estadio IIA (T3N0M0). Em consulta de grupo multidisciplinar foi decidido, tendo em conta o quadro clínico, que manteria vigilância oncológica sem necessidade de quimioterapia. A Maria foi orientada para consulta de oncologia e consulta de cirurgia geral, onde tem sido acompanhada.

Após alta do internamento tem tido consultas mensais com a sua médica de família para renovação de certificado de incapacidade temporária para o trabalho, por ainda não se encontrar apta para trabalhar, uma vez que mantém dejeções diarreicas frequentes na sequência da cirurgia, bem como alguns episódios de pouchite e estenose da bolsa, com necessidade de internamento, que resolveram com dilatação endoscópica.

\section{COMENTÁRIO}

Este caso clínico demonstra a importância de co- nhecer a história familiar dos utentes. Através do genograma familiar foi possível perceber a existência de um padrão de transmissão hereditária de patologia do trato gastrointestinal. O tempo que leva a realizar um genograma constitui uma limitação deste instrumento, tendo em consideração o agendamento de consultas de 20 em 20 minutos, pelo que o mesmo não foi realizado na primeira consulta. ${ }^{3}$ Foi, no entanto, abordada a história familiar no primeiro contacto com a utente, que mencionou todos os familiares que apresentavam problemas gastrointestinais.

Apesar de a Maria ter em Portugal Continental vários familiares com o diagnóstico de PAF, desconhecia que esta doença estivesse na origem dos casos de patologia gastrointestinal que ocorriam na família e que o seu pai deveria ter feito o rastreio para esta patologia.

O olhar atento da enfermeira de família e a comunicação da mesma com a médica permitiram uma orientação mais rápida e eficaz do caso.

A presença de dor abdominal com sinais de alarme (perda de peso superior a 5\% num período tempo inferior a três meses; história familiar de patologia gastrointestinal; sintomas noturnos; presença de defesa na palpação do abdómen e sinal de blumberg presente) levaram à referenciação da utente para o serviço de urgência, dada a necessidade de realizar exames complementares de diagnóstico com urgência para esclarecer o quadro abdominal. ${ }^{4}$

Após a alta do serviço de urgência e a observação em consulta de medicina interna decorreu ainda algum tempo até à realização da colonoscopia total, exame que continua a não estar acessível nos cuidados de saúde primários na ilha de São Miguel, estando o seu pedido pelo médico de família dependente da referenciação do utente a consulta hospitalar.

O tratamento cirúrgico ao qual a Maria foi submetida (colectomia total com anastomose íleo-retal baixa com bolsa em J) é uma das principais opções de tratamento para a forma clássica da PAF, mas implica vigilância endoscópica anual da bolsa pelo risco aumentado de carcinomas e adenomas da mesma. ${ }^{2}$ Pode ter como complicações dejeções diarreicas frequentes, estenose da bolsa e pouchite, as quais a Maria tem apresentado após a intervenção e que interferem na sua qualidade de vida, impedindo-a de trabalhar por enquanto. Devido ao trânsito intestinal rápido apresenta- 
do pela Maria deverão também vigiar-se possíveis défices de vitamina B12.5-8

Relativamente ao seguimento da Maria, nos utentes com o diagnóstico de PAF é importante convocar os familiares em risco de apresentar esta síndroma, nomeadamente os de primeiro grau, bem como alertar para a importância do rastreio através de retosigmoidoscopia ou de colonoscopia anual a partir dos 10-12 anos. ${ }^{9-10}$ Existem ainda testes genéticos que devem ser realizados sempre que possível a partir dos 10-12 anos, mas que podem apresentar falsos negativos pelo que, mesmo que o teste seja negativo, deve programar-se uma vigilância sigmoidoscópica a cada três a cinco anos até aos 40 anos de idade.,

A probabilidade de transmissão desta síndroma para os filhos deve ser explicada e deve relembrar-se a importância da realização da consulta pré-concecional, assim como a referenciação para a consulta de diagnóstico pré-natal quando a Maria quiser engravidar.

No acompanhamento de utentes com PAF é importante ter presente a necessidade de vigiar o aparecimento de outros tipos de patologia, como tumor desmoide, neoplasia tiroideia e hipertrofia congénita do epitélio da retina, motivo pelo qual está recomendada a endoscopia digestiva alta a cada seis meses a quatro anos, de acordo com o estadio de Spigelman de polipose duodenal, a partir dos 25-30 anos; a ecografia tiroideia anual a partir dos 25-30 anos e o exame oftalmológico anual. No caso de surgirem sintomas ou fatores de risco para tumor desmoide, como história familiar de tumor desmoide, mutação para além do codão 1444 de Adenomatous Polyposis Coli, massa abdominal palpável ou sintomas sugestivos de obstrução de órgão abdominal, a tomografia computorizada ou a ressonância magnética abdomino-pélvica estão recomendadas. ${ }^{5}$

Finalmente, é de valorizar o facto de a Maria ter aceitado o convite para apresentar o seu caso peculiar aos alunos de medicina da universidade local.

\section{REFERÊNCIAS BIBLIOGRÁFICAS}

1. Ternent CA, Thorson AG. Síndromes da polipose adenomatosa familiar [homepage]. BM] Best Practice; 2016 [updated 2018 Feb; revised 2020 Jan]. Available from: https://bestpractice.bmj.com/topics/pt-br/652

2. Freitas D. Pólipos e síndrome de polipose do cólon e recto. In: Freitas
D, editor. Doenças do aparelho digestivo. $2^{\text {a }}$ ed. Lisboa: AstraZeneca; 2002. p. 429-33. ISBN 9728157886

3. Rebelo L. Genograma familiar: o bisturi do médico de família [The genogram: the scalpel of the family doctor]. Rev Port Clin Geral. 2007;23(3):309-17. Portuguese

4. Coda S, Thillainayagam AV. Symptoms and signs of lower gastrointestinal disease. Medicine. 2015;43(3):146-52.

5. Chung DC. Familial adenomatous polyposis: screening and management of patients and families. UpToDate [Internet]; 2013 [updated 2018 Oct 11; revised 2020 Jan]. Available from: https://www.uptodate.com/contents/familial-adenomatous-polyposis-screening-and-management-of-patients-and-families? source=search_result \&search=familialadenomatous\%20polyposis\%20screening\%20and\%20management\%20of\%20patients\%20and\%20families\&selectedTitle=2 150

6. Friederich $P$, van Heumen BW, Nagtegaal ID, Berkhout $M$, van Krieken $\mathrm{JH}$, Peters $\mathrm{WH}$, et al. Increased epithelial cell proliferation in the ileal pouch mucosa of patients with familial adenomatous polyposis. Virchows Arch. 2007;451(3):659-67.

7. Lynch PM. Jurisprudential considerations in the evaluation and screening of high-risk patients. In: Rozen P, Winawer SJ, editors. Secondary prevention of colorectal cancer: an international perspective. Basel: Karger; 1986. p. 55-63.

8. Leal RF, Ayrizono ML, Coy CS, Fagundes JJ, Góes JR. Complicações imediatas e tardias após cirurgia de reservatório ileal na polipose adenomatosa familiar [Short-term and long-term postoperative complications after ileal pouch-anal anastomosis in familial adenomatous polyposis]. Arq Gastroenterol. 2008;45(2):106-10. Portuguese

9. Ramsey SD, Grady WM. Screening for colorectal cancer in patients with a family history of colorectal cancer or advanced polyp. UpToDate [Internet]; 2015 [updated 2019 Mar 25; revised 2020 Jan]. Available from: https://www.uptodate.com/contents/screening-for-colorectal-cancerin-patients-with-a-family-history-of-colorectal-cancer-or-advancedpolyp

10. Chung DC. Clinical manifestations and diagnosis of familial adenomatous polyposis. UpToDate [Internet]; 2013 [updated 2019 Nov 25; revised 2020 Jan]. Available from: https://www.uptodate.com/contents/clinical-manifestations-and-diagnosis-of-familial-adenomatouspolyposis?source=search_result\&search=familialadenomatous $\% 20$ polyposis\%20screening\%20and\%20management\%20of\%20patients\%2 Oand\%20families\&selectedTitle $=4 \sim 150$

\section{CONFLITO DE INTERESSES}

Os autores declaram não ter quaisquer conflitos de interesse.

\section{ENDEREÇO PARA CORRESPONDÊNCIA}

Sofia Correia

E-mail: sofia.fj.correia@gmail.com

https://orcid.org/0000-0003-2904-7227

Recebido em 21-12-2017

Aceite para publicação em 03-10-2018 


\section{ABSTRACT}

\section{FAMILIAL ADENOMATOUS POLYPOSIS: A CASE REPORT}

Introduction: The familial adenomatous polyposis syndrome (FAP), in its classic form, is autosomal dominant. The affected individuals may remain asymptomatic for several years, so it's important to know their family history and maintain a certain degree of clinical suspicion. Therefore, this case intends to reinforce the role of the family doctor in the approach of this rare pathology and emphasize the need for perfectly coordinated teamwork and health care, bearing in mind the difficulties of a timely diagnosis.

Case description: A 20-year-old woman was observed by her family doctor for vomiting, frequent diarrhea and weight loss of $7 \mathrm{~kg}$ in the last two weeks. She had pain and rebound tenderness on palpation of the peri-umbilical region and left hypochondrium. Through the clinical interview and realization of the family genogram, it was verified the existence of a pattern of hereditary transmission of the pathology of the gastrointestinal tract. She was sent to the emergency department of the hospital, where the existence of surgical and gynecological pathology was excluded, having medical release but referred for internal medicine consultation. After a complete colonoscopy, she was admitted for surgical resection of adenocarcinoma of the sigmoid colon.

Comment: This case highlights both the importance of the genogram and the temporal limitation on its application by the family doctor. It also reinforces the importance of integrating and coordinating health care in this pathology. The family doctor should know the possible complications of the surgical intervention, as well as other pathologies associated with FAP and what kind of follow-up should be done in these patients. In this case, it is also necessary to take into account the need for screening of other affected relatives and the importance of pre-conception consultation and/or prenatal diagnosis.

Keywords: Familial Adenomatous Polyposis; Genogram; Abdominal Pain; General Practice. 